\title{
Estudo colaborativo para avaliação dos teores de matéria mineral em alimentos
}

\author{
Collaborative study to evaluate the mineral content in feeds
}

\author{
SOUZA, Marjorrie Augusto de ${ }^{1}$; DETMANN, Edenio ${ }^{1 *}$; BATISTA, Erick Darlisson ${ }^{1}$; \\ FRANCO, Marcia de Oliveira ; VALADARES FILHO, Sebastião de Campos ${ }^{1}$; PINA, \\ Douglas Santos ${ }^{2}$; ROCHA, Gabriel Cipriano ${ }^{1}$
}

\footnotetext{
${ }^{1}$ Universidade Federal de Viçosa, Departamento de Zootecnia, Viçosa, Minas Gerais, Brasil.

${ }^{2}$ Universidade Federal de Mato Grosso, Departamento de Zootecnia, Sinop, Mato Grosso, Brasil.

*Endereço para correspondência: detmann@ufv.br
}

\section{RESUMO}

Objetivou-se conduzir estudo colaborativo para avaliação do teor de matéria mineral em alimentos em sete laboratórios de análise de alimentos de instituições integrantes do Instituto Nacional de Ciência e Tecnologia de Ciência Animal (INCT-CA). Avaliaram-se seis alimentos: feno de capim-braquiária, cana-deaçúcar, silagem de milho grão, farelo de soja, milho e polpa cítrica. Solicitou-se a cada laboratório que as amostras fossem analisadas por seis dias, consecutivos ou não, sendo realizadas três repetições por amostra por dia de avaliação. Foi observado variação entre laboratórios, dias de análise e efeito de interação de material e laboratório. A variação dos resultados entre laboratórios correspondeu de 33,5 a $78,2 \%$ da variação aleatória total. Verificou-se que a repetibilidade representou de 8,5 a $51,6 \%$ da reprodutibilidade. Observou-se razões de Horwitz superiores a dois para três dos materiais avaliados. Foi constatado que $42,9 \%$ das avaliações apresentaram variações das estimativas em função do dia no qual a análise foi realizada. Também foram verificadas diferenças entre procedimentos de avaliação de matéria mineral em função dos diferentes laboratórios. Os teores de matéria mineral estimados pelos laboratórios participantes deste estudo apresentam baixa reprodutibilidade, o que é agravado pela presença de efeito de interação do material avaliado e do laboratório.

Palavras-chave: análise de alimentos, cinzas, métodos de análises

\section{SUMMARY}

The objective was to conduct a collaborative study to evaluate the mineral matter contents in feeds in seven feed analysis laboratories from institutions linked to the National Institute of Science and Technology in Animal Science (INCT-CA). Six feeds were evaluated: signal grass hey, sugarcane, corn silage, soybean meal, corn grain and citrus pulp. It was asked to each laboratory that the samples were analyzed for six days, consecutive or not, with three replicates per sample per day of evaluation. Variations were observed among laboratories, days of analysis and interaction effect of material and laboratory. The variation of results among laboratories accounted from 33.5 to $78.2 \%$ of the total random variation. It was verified that the repeatability represented 8.5 to $51.6 \%$ of reproducibility. It was noticed Horwitz ratios greater than 2 for three materials tested. It was found that $42.9 \%$ of the assessments show variations of the estimates as a function of days in which analysis was performed. Differences were also observed between procedures to assess mineral matter according to the different laboratories. The mineral matter contents estimate by each laboratory in this study have low reproducibility, which is aggravated by the interaction effect of material and laboratory.

Keywords: analytical methods, ash, feed analysis 


\section{INTRODUÇÃO}

A matéria mineral (MM) é constituída pelo resíduo inorgânico obtido após a queima da matéria orgânica, a qual é convertida em $\mathrm{CO}_{2}, \mathrm{H}_{2} \mathrm{O}$ e $\mathrm{NO}_{2}$, e eliminada em conjunto com as substâncias voláteis decompostas pelo calor.

O método consiste basicamente na incineração do alimento em altas temperaturas (normalmente de 500 a $600^{\circ} \mathrm{C}$ ), por tempo suficiente para que ocorra combustão total da matéria orgânica (SILVA \& QUEIROZ, 2002; CECCHI, 2003).

Sendo a matéria seca total do alimento formada pelas frações orgânica e inorgânica, o teor de MM em alimentos atua como estimador indireto do conteúdo de componentes orgânicos totais. Adicionalmente, o conhecimento do teor de MM se faz necessário para se conhecer a proporção dos componentes quantificados por diferença em alimentos, como o extrativo não nitrogenado e os carboidratos não fibrosos.

Desta forma, faz-se necessária a avaliação dos procedimentos laboratoriais e das estimativas de MM produzidas em diferentes materiais e por diferentes laboratórios, buscando-se identificar métodos mais adequados e padronizar os procedimentos entre laboratórios de análise de alimentos no país.

Objetivou-se conduzir estudo colaborativo para avaliação do teor de MM em diferentes amostras de alimentos em sete laboratórios de análise de alimentos de instituições integrantes do Instituto Nacional de Ciência e Tecnologia de Ciência Animal (INCT-CA).

\section{MATERIAL E MÉTODOS}

As avaliações foram conduzidas em sete laboratórios de análise de alimentos de instituições componentes do INCT-CA: Universidade Federal de Viçosa, Viçosa-MG; Escola de Veterinária da Universidade Federal de Minas Gerais, Belo Horizonte-MG; Universidade Federal de Lavras, Lavras-MG; Universidade Estadual de Santa Cruz, Ilhéus-BA; Universidade Estadual Paulista Júlio de Mesquita, JaboticabalSP; Universidade Federal de Mato Grosso, Cuiabá-MT; e Universidade Federal de Mato Grosso, Sinop-MT.

Foram avaliadas amostras de seis diferentes alimentos: feno de capimbraquiária, cana-de-açúcar, silagem de milho grão, farelo de soja, milho e polpa cítrica. Em adição, foi enviado composto padrão para aferição da exatidão das estimativas (celite; Sigma C-8656).

As amostras de volumosos úmidos foram secas em estufa com ventilação forçada $\left(60^{\circ} \mathrm{C}\right)$ e, em conjunto com as demais amostras, processadas em moinho de facas $(1 \mathrm{~mm})$, acondicionadas em sacos plásticos e enviadas sem identificação, juntamente com o padrão, aos laboratórios.

Na oportunidade do envio, solicitou-se a cada laboratório que amostras fossem analisadas por seis dias, consecutivos ou não, sendo realizadas três repetições por amostra por dia de avaliação.

Os resultados foram solicitados com base na amostra seca ao ar, uma vez que a avaliação de resultados com correção para o teor de matéria seca de cada amostra entre laboratórios pode apresentar erro oriundo de dois procedimentos (MERTENS, 2003).

Em conjunto com as amostras, enviouse questionário para elucidação dos principais procedimentos e parâmetros 
analíticos quantitativos e qualitativos adotados em cada laboratório.

Após o recebimento dos dados, os teores de $\mathrm{MM}$ referentes às amostras foram avaliados de acordo com modelo: $Y_{i j k l}=\mu+L_{i}+M_{j}+L M_{i j}+D_{(i j) k}+\varepsilon_{(i j k) l}$ (1); em que: $\mathrm{Y}_{\mathrm{ijkl}}=$ teor de MM (\%) obtida no laboratório i, no material j, na repetição 1 avaliada no dia de análise k; $\mu=$ constante geral; $L_{i}=$ efeito do laboratório i (aleatório); $\mathrm{M}_{\mathrm{j}}=$ efeito do material $\mathrm{j}$ (fixo); $\mathrm{LM}_{\mathrm{ij}}=$ efeito de interação do laboratório $i$ e material $\mathrm{j}$ (aleatório); $\mathrm{D}_{(\mathrm{ij}) \mathrm{k}}=$ efeito do dia de análise $\mathrm{k}$, aninhado à combinação entre o laboratório i e o material j (aleatório); e $\varepsilon_{(\mathrm{ijk}) 1}=$ erro aleatório, associado a cada mensuração, pressuposto NID $\left(0 ; \sigma_{\varepsilon}^{2}\right)$.

Por intermédio do método dos momentos, utilizando-se os fundamentos do procedimento de Hicks modificado (BARBIN, 1993), definiram-se as esperanças de quadrados médios da análise de variância (Tabela 1).

As observações classificadas como outliers, foram eliminadas do conjunto de dados, sendo estas definidas quando seu desvio em relação à média das avaliações do material, dentro do laboratório, superasse em três vezes o valor do desvio padrão residual.

A partir das esperanças de quadrados médios apresentadas na Tabela 1 foi estimada a repetibilidade (r) e a reprodutibilidade $(\mathrm{R})$ dos teores de $\mathrm{MM}$, dadas por (MERTENS, 2003):

$$
\begin{aligned}
& r=\hat{\sigma}_{\varepsilon}^{2} \\
& R=\hat{\sigma}_{\varepsilon}^{2}+\hat{\sigma}_{L}^{2}
\end{aligned}
$$

Tabela 1. Esperança de quadrados médios para modelo destinado à análise global dos dados

\begin{tabular}{lc}
\hline Fonte de Variação ${ }^{1}$ & \multicolumn{1}{c}{$\mathrm{E}(\mathrm{QM})^{2}$} \\
\hline $\mathrm{L}$ & $\sigma_{\varepsilon}^{2}+\mathrm{k}_{1} \times \sigma_{\mathrm{D} / \mathrm{L} \times \mathrm{M}}^{2}+\mathrm{k}_{2} \times \sigma_{\mathrm{L} \times \mathrm{M}}^{2}+\mathrm{k}_{4} \times \sigma^{2} \mathrm{~L}$ \\
$\mathrm{M}$ & $\sigma_{\varepsilon}^{2}+\mathrm{k}_{1} \times \sigma_{\mathrm{D} / \mathrm{L} \times \mathrm{M}}+\mathrm{k}_{2} \times \sigma_{\mathrm{L} \times \mathrm{M}}+\mathrm{k}_{3} \times \phi_{\mathrm{M}}$ \\
$\mathrm{L} \times \mathrm{M}$ & $\sigma_{\varepsilon}^{2}+\mathrm{k}_{1} \times \sigma_{\mathrm{D} / \mathrm{L} \times \mathrm{M}}+\mathrm{k}_{2} \times \sigma_{\mathrm{L} \times \mathrm{M}}^{2}$ \\
$\mathrm{D} / \mathrm{L} \times \mathrm{M}$ & $\sigma_{\varepsilon}^{2}+\mathrm{k}_{1} \times \sigma_{\mathrm{D} / \mathrm{L} \times \mathrm{M}}$ \\
Resíduo & $\sigma_{\varepsilon}^{2}$ \\
\hline${ }^{1} \mathrm{~L}=$ laboratório; $\mathrm{M}=$ material; $\mathrm{L} \times \mathrm{M}=$ interação laboratório $\times$ material, $\mathrm{D} / \mathrm{L} \times \mathrm{M}=$ dia aninhado às \\
combinações entre laboratório e material. \\
${ }^{2} \sigma_{\varepsilon}^{2}, \sigma_{\mathrm{D} / \mathrm{L} \times \mathrm{M},}, \sigma_{\mathrm{L} \times \mathrm{M},}^{2} \sigma^{2}=$ variâncias associadas aos efeitos do erro (repetibilidade), de dia aninhado às \\
combinações entre laboratório e material, da interação de laboratório e material e de laboratório, \\
respectivamente; $\phi_{\mathrm{M}}=$ função quadrática associada ao efeito de material.
\end{tabular}

Quando o efeito de interação de material analisado e laboratório foi significativo, procedeu-se a nova avaliação, na qual considerou-se cada material isoladamente. Esclarece-se que na presença de interação, tanto a reprodutibilidade como a repetibilidade estimadas a partir dos componentes de variância obtidos na equação (1) não podem ser utilizadas para representar $o$ comportamento de cada material em geral. Como são avaliados efeitos aleatórios não é possível realizar o desdobramento em um único modelo. Cabe ressaltar, que efeitos aleatórios não constituem parâmetros da população, mas sim constituem variáveis aleatórias contínuas. Desta forma, um componente de variância comum para laboratório não poderá ser usado para a avaliação de cada um dos materiais. Como o desdobramento em 
um único modelo é impossível neste caso, a estimação de componentes de variâncias individuais só é possível com o fracionamento do banco de dados. Desta forma, o desdobramento foi realizado utilizando-se um modelo para avaliação da variação de cada material considerando-se os diferentes laboratórios: $Y_{i j k}=\mu+L_{i+} D_{(i) j+} \varepsilon_{(i j) k} \quad$ (4); em que: $\mathrm{Y}_{\mathrm{ijk}}=$ teor de MM (\%) obtida no laboratório $\mathrm{i}$, na repetição $\mathrm{k}$ avaliada no dia de análise $\mathrm{j} ; \mu=$ constante geral; $\mathrm{L}_{\mathrm{i}}=$ efeito do laboratório i (aleatório); $\mathrm{D}_{(\mathrm{i}) \mathrm{j}}=$ efeito do dia de análise $\mathrm{j}$ aninhado ao laboratório i (aleatório); e $\varepsilon_{(\mathrm{ij}) \mathrm{k}}=$ erro aleatório, associado a cada mensuração, pressuposto NID $\left(0 ; \sigma_{\varepsilon}^{2}\right)$.

O modelo descrito em (4) foi também utilizado para avaliação da recuperação da MM do padrão.

As esperanças de quadrados médios para o modelo descrito em (4) são descritas na Tabela 2. As estimativas de repetibilidade e reprodutibilidade para cada material foram obtidas de forma similar ao descrito nas Equações (2) e (3).

Para a situação descrita na Tabela 2, procedeu-se à avaliação das estimativas de reprodutibilidade esperada e da razão de Horwitz (HORWITZ et al., 1990) por intermédio das equações:

$$
\begin{aligned}
& R p=\frac{\hat{\sigma}_{R}}{\bar{X}} \\
& \hat{\sigma}_{R}=\sqrt{R} \\
& R p e=2 \exp (1-0,5 \log c) \\
& R H=\frac{R p}{R p e}
\end{aligned}
$$

em que: $\mathrm{Rp}=$ reprodutibilidade padronizada em função da média; Rpe = reprodutibilidade padronizada em função da média estimada pelo método de Horwitz; c = concentração média de MM (g/g); e RH = razão de Horwitz.

Tabela 2. Esperanças de quadrados médios para o modelo destinado à análise de cada material em função dos diferentes laboratórios (Equação 4)

\begin{tabular}{lc}
\hline Fonte de Variação $^{1}$ & $\mathrm{E}(\mathrm{QM})^{2}$ \\
\hline $\mathrm{L}$ & $\sigma_{\varepsilon}^{2}+\mathrm{k}_{1} \times \sigma_{\mathrm{D} / \mathrm{L}}^{2}+\mathrm{k}_{2} \times \sigma_{\mathrm{L}}^{2}$ \\
$\sigma_{\varepsilon}^{2}+\mathrm{k}_{1} \times \sigma_{\mathrm{D} / \mathrm{L}}^{2}$ \\
$\mathrm{D} / \mathrm{L}$ & $\sigma_{\varepsilon}^{2}$ \\
Resíduo & \\
${ }^{1} \mathrm{~L}=$ laboratório; $\mathrm{D} / \mathrm{L}=$ dia aninhado ao laboratório. \\
${ }^{2} \sigma_{\varepsilon}^{2}, \sigma_{\mathrm{D} / \mathrm{L}}, \sigma_{\mathrm{L}}^{2}=$ variâncias associadas aos efeitos do erro (repetibilidade), de dia aninhado ao \\
laboratório e de laboratório, respectivamente.
\end{tabular}

Quando o efeito de dia de análise foi significativo, procedeu-se ao desdobramento das avaliações conforme explicações apresentadas anteriormente, com a avaliação do comportamento de cada material em cada laboratório por intermédio do modelo: $Y_{i j}=\mu+D_{i+} \varepsilon_{(i) j}(9)$; em que: $\mathrm{Y}_{\mathrm{ij}}=$ teor de MM (\%) obtida na repetição $\mathrm{j}$ avaliada no dia de análise $\mathrm{i} ; \mu$ $=$ constante geral; $\mathrm{D}_{\mathrm{i}}=$ efeito do dia de análise i (aleatório); e $\varepsilon_{(\mathrm{i}) \mathrm{j}}=$ erro aleatório, associado a cada mensuração, pressuposto NID $\left(0 ; \sigma_{\varepsilon}^{2}\right)$.

Por intermédio do método dos momentos (BARBIN, 1993), definiram-se as esperanças de quadrados médios associadas ao modelo descrito em (9) (Tabela 3).

A variação aleatória total associada ao modelo descrito em (9) foi dada por: $\hat{\sigma}$ $\hat{\sigma}_{T}^{2}=\hat{\sigma}_{D}^{2}+\hat{\sigma}_{\varepsilon}^{2}$ 
Todos os procedimentos estatísticos foram conduzidos por intermédio do programa SAS (Statisical Analysis System, versão 9.2), adotando-se 0,01 como nível crítico de probabilidade para o erro tipo I. As comparações entre médias fornecidas por diferentes laboratórios foram conduzidas utilizando-se o critério de Scheffé. Por outro lado, a avaliação da recuperação de MM a partir do padrão de acordo com os laboratórios foi realizada utilizando-se as propriedades de intervalos de confiança $(1-\alpha=0,99)$.

Tabela 3. Esperanças de quadrados médios para o modelo destinado à análise de cada material em cada laboratório em função dos dias de análise (Equação 9)

\begin{tabular}{lc}
\hline Fonte de Variação $^{1}$ & $\mathrm{E}(\mathrm{QM})^{2}$ \\
\hline $\mathrm{D}$ & $\sigma_{\varepsilon}^{2}+\mathrm{k}_{1} \times \sigma_{\mathrm{D}}^{2}$ \\
Resíduo & $\sigma_{\varepsilon}^{2}$ \\
\hline${ }^{1} \mathrm{D}=$ dia. $^{2} \sigma_{\varepsilon}^{2}, \sigma_{\mathrm{D}}^{2}=$ variâncias associadas aos efeitos do erro e de dia de análise, respectivamente.
\end{tabular}

\section{RESULTADOS E DISCUSSÃO}

$\mathrm{Na}$ avaliação do padrão, não se observou significância $(\mathrm{P}>0,01)$ para os componentes de variância. Isto indica que os teores obtidos de MM são independentes do dia em que a análise foi realizada e do laboratório onde as análises foram realizadas, $o$ que constitui condição teórica ideal (Tabela 4). Neste caso, a reprodutibilidade foi constituída única e exclusivamente pela variação entre repetições (repetibilidade). Sob estas condições, em todos os laboratórios verificou-se recuperação completa $(\mathrm{P}>0,01)$ da $\mathrm{MM}$ oriunda do padrão (Tabela 5).

Contudo, de forma contrária ao verificado para o padrão, observou se que todos os componentes de variância da avaliação global das amostras foram significativos $(\mathrm{P}<0,01)$. Isto indica que, além das diferenças entre laboratórios, os teores estimados de MM se mostram dependentes do dia em que a analise foi realizada e que materiais diferentes produziram estimativas diferentes em função do laboratório no qual a análise foi realizada (Tabela 6). Este comportamento talvez possa ser explicado pela interação complexa dos componentes orgânicos e minerais nas amostras, o que demandaria mais do método em comparação à avaliação de celite. Logo, os resultados obtidos com o padrão não parecem refletir fidedignamente as condições de análise dos laboratórios avaliados.

Tabela 4. Estimativas de componentes de variância do modelo global (padrão; Equação 4)

\begin{tabular}{lccc}
\hline Item & Estimativa & Valor relativo (\%) & Valor-P \\
\hline$\sigma^{2} \mathrm{~L}$ & $<0,0001$ & 0,0 & 0,0902 \\
$\sigma^{2}{ }_{\mathrm{D} / \mathrm{L}}$ & $<0,0001$ & 0,0 & 0,9993 \\
Repetibilidade $\left(\sigma_{\varepsilon}^{2}\right)$ & 0,0006 & 100,0 & - \\
Variância Total & 0,0006 & 100,0 & - \\
Reprodutibilidade & 0,0006 & - & - \\
$\mathrm{r} / \mathrm{R}^{1}$ & $\approx 1$ & - & - \\
\hline
\end{tabular}

${ }^{1}$ Razão repetibilidade/reprodutibilidade. 
Tabela 5. Recuperação do padrão (g/g) pelos diferentes laboratórios

\begin{tabular}{lc}
\hline Item & Celite \\
\hline Teor Esperado $^{1}$ & 99,96 \\
\hline Laboratório 1 & 1,0012 \\
Laboratório 2 & 1,0010 \\
Laboratório 3 & 1,0018 \\
Laboratório 4 & 0,9896 \\
Laboratório 5 & 1,0019 \\
Laboratório 6 & 1,0017 \\
Laboratório 7 & 1,0047 \\
\hline
\end{tabular}

${ }^{1} \mathrm{O}$ teor esperado de $\mathrm{MM}$ no celite foi assumido como $100 \%$, com base na matéria seca. Este valor foi corrigido para o teor de umidade no material, o qual foi estimado por intermédio do método de Karl Fischer (BRUTTEL \& SCHLINK, 2006).

Tabela 6. Estimativas de componentes de variância do modelo global (amostras; Equação 1)

\begin{tabular}{lccc}
\hline Item & Estimativa & Valor relativo (\%) & Valor-P \\
\hline$\sigma^{2}{ }_{\mathrm{L}}$ & 0,1348 & 39,3 & $<0,0001$ \\
$\sigma_{\mathrm{LxM}}$ & 0,0835 & 24,4 & $<0,0001$ \\
$\sigma_{\mathrm{D} / \mathrm{LxM}}^{2}$ & 0,0613 & 17,9 & $<0,0001$ \\
Repetibilidade $\left(\sigma_{\varepsilon}^{2}\right)$ & 0,0632 & 18,4 & - \\
Variância Total & 0,3428 & 100,0 & - \\
Reprodutibilidade & 0,1980 & - & - \\
$\mathrm{r} / \mathrm{R}^{1}$ & 0,3192 & - & - \\
Média & 5,6439 & - & - \\
\hline
\end{tabular}

${ }^{1}$ Razão repetibilidade/reprodutibilidade.

De forma geral, assume-se como não aceitável a ocorrência de efeito de interação de material e laboratório (MERTENS, 2003). A ocorrência deste indica que os possíveis erros sistemáticos imputados a um dado material em determinado laboratório serão distintos daqueles observados em outro laboratório. A isto se adiciona o fato de que os erros sistemáticos imputados por um laboratório se manifestarão de forma diferenciada em função do material analisado. Isto atribui elemento de confundimento aos cotejamentos das características químicas de alimentos e outros materiais, como fezes, sobras e digestas; o que, consequentemente, pode comprometer a comparação entre características obtidas em ensaios de digestão.

A interação de material e laboratório é corroborada pela avaliação comparativa das médias obtidas nos diferentes alimentos, na qual se observou alta variação nas diferenças entre laboratórios, não havendo padrão definido nos perfis de comparações múltiplas. Verificou-se que as diferenças entre laboratórios variaram de 0,45 ponto percentual para o milho a 2,61 pontos percentuais para a polpa cítrica (Tabela 7).

Analisando-se os materiais isoladamente, observou-se grande variação na reprodutibilidade (Figura 1), o que parece mais uma vez corroborar o efeito de interação de laboratório e material (Tabela 7). 
Tabela 7. Comparação entre médias produzidas pelos diferentes laboratórios para feno, cana-de-açúcar, silagem de milho (SM), farelo de soja (FS), milho, polpa cítrica $(\mathrm{PC})$

\begin{tabular}{lcccccc}
\hline \multirow{2}{*}{ Laboratório } & \multicolumn{7}{c}{ Material $^{1,2}$} \\
\cline { 2 - 7 } & Feno & Cana & SM & FS & Milho & PC \\
\hline 1 & $7,28^{\mathrm{c}}$ & $6,77^{\mathrm{bc}}$ & $5,53^{\mathrm{b}}$ & $5,69^{\mathrm{de}}$ & $1,06^{\mathrm{cd}}$ & $5,92^{\mathrm{d}}$ \\
2 & $7,45^{\mathrm{bc}}$ & $7,03^{\mathrm{ab}}$ & $5,66^{\mathrm{ab}}$ & $5,99^{\mathrm{bc}}$ & $1,09^{\mathrm{cd}}$ & $7,15^{\mathrm{ab}}$ \\
3 & $7,88^{\mathrm{a}}$ & $7,42^{\mathrm{a}}$ & $5,67^{\mathrm{ab}}$ & $6,36^{\mathrm{a}}$ & $1,21^{\mathrm{b}}$ & $6,84^{\mathrm{bc}}$ \\
4 & $6,69^{\mathrm{d}}$ & $6,21^{\mathrm{c}}$ & $5,17^{\mathrm{c}}$ & $5,45^{\mathrm{e}}$ & $0,94^{\mathrm{d}}$ & $4,96^{\mathrm{e}}$ \\
5 & $7,64^{\mathrm{ab}}$ & $7,44^{\mathrm{a}}$ & $5,78^{\mathrm{ab}}$ & $6,12^{\mathrm{ab}}$ & $1,14^{\mathrm{bc}}$ & $6,73^{\mathrm{c}}$ \\
6 & $7,47^{\mathrm{bc}}$ & $7,14^{\mathrm{ab}}$ & $5,68^{\mathrm{ab}}$ & $5,81^{\mathrm{cd}}$ & $1,00^{\mathrm{cd}}$ & $7,39^{\mathrm{a}}$ \\
7 & $7,89^{\mathrm{a}}$ & $7,33^{\mathrm{ab}}$ & $5,85^{\mathrm{a}}$ & $6,35^{\mathrm{a}}$ & $1,39^{\mathrm{a}}$ & $7,57^{\mathrm{a}}$ \\
$\mathrm{EPM}^{\mathrm{ATM}}{ }^{3}$ & 0,12 & 0,13 & 0,08 & 0,10 & 0,07 & 0,17 \\
\hline
\end{tabular}

${ }^{1}$ Médias na coluna, seguidas por letras diferentes, são diferentes pelo teste de Scheffé $(\mathrm{P}<0,01)$.

${ }^{2}$ ATM, amplitude total entre médias.

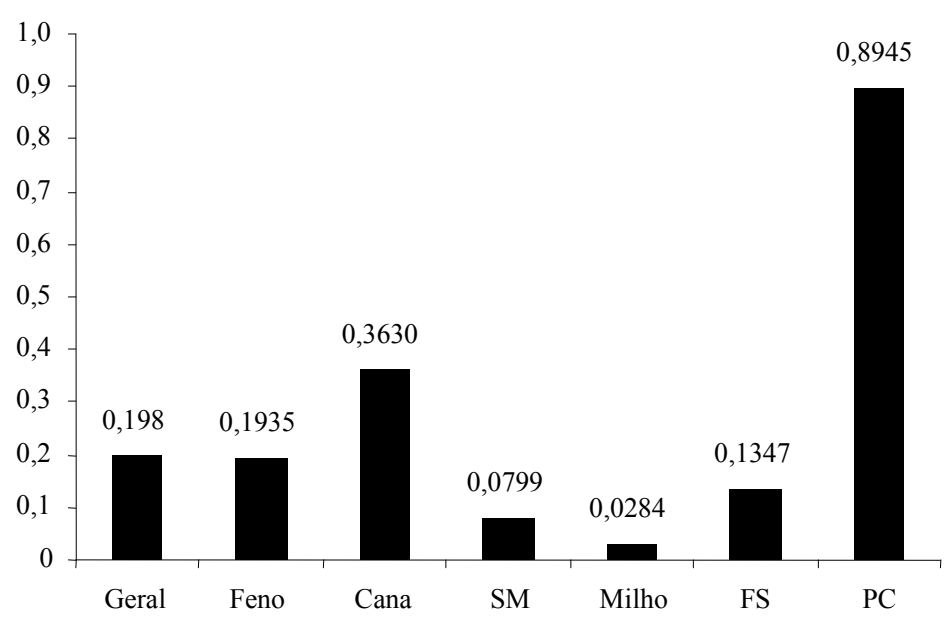

Figura 1. Reprodutibilidade $\left[(\%)^{2}\right]$ para os diferentes materiais analisados

Na avaliação individual de cada material, observou-se que a variação dos resultados entre laboratórios correspondeu de 33,5 a $78,2 \%$ da variação aleatória total. Adicionalmente, verificou-se que a repetibilidade (variação entre repetições) representou de 8,5 a $51,6 \%$ da reprodutibilidade (Tabelas 8 e 9). $\mathrm{O}$ comportamento destes percentuais reitera que diferenças entre laboratórios existem e são significativas quando se analisa os materiais individualmente, correspondendo ao fator preponderante das diferenças entre os teores de MM.
A avaliação da reprodutibilidade, a qual é dada pela soma da repetibilidade e da variação entre laboratórios, apresentou-se em patamares considerados aceitáveis para três dos seis alimentos avaliados. Contudo, razões de Horwitz $(\mathrm{RH})$ superiores a 2 foram verificadas para os demais materiais avaliados (Tabelas 8 e 9). Este parâmetro é dado pela razão entre a reprodutibilidade observada e a reprodutibilidade que deveria ser esperada considerando-se a concentração média do componente analisado (HORWITZ et al., 1990).

Pode se concluir que as avaliações não podem ser consideradas adequadamente 
reprodutíveis para a metade dos materiais, pois RH superiores a 2 sugerem que o método avaliado é inaceitável com respeito à sua reprodutibilidade (MERTENS, 2003). Assim, os valores estimados de RH indicam que a reprodutibilidade é essencialmente comprometida por diferenças nas condições de análise.

As repetibilidades padronizadas (expressas como percentuais das médias) foram estimadas a partir dos dados expressos nas Tabelas 8 e 9 .

Foram verificados valores de 2,$60 ; 6,14$; 3,$44 ; 2,90 ; 9,36$ e $4,15 \%$ para feno, canade-açúcar, silagem de milho, farelo de soja, milho e polpa cítrica, respectivamente, indicando baixos valores relativos da variação entre repetições. Logo, sendo a reprodutibilidade dada pela soma da variação entre repetições (repetibilidade) e entre laboratórios, os altos valores de $\mathrm{RH}$ (Tabelas 8 e 9) parecem ter sido causados pela elevada variação inter-laboratorial. Considerando-se as informações das tabelas 8 e 9, percebeu-se que ocorreu efeito significativo do dia de análise $(\mathrm{P}<0,01)$ sobre as estimativas de MM para todos os materiais exceto para cana-deaçúcar. A avaliação isolada deste efeito para cada material analisado em cada laboratório indicou que $42,9 \%$ das avaliações apresentaram variações significativas $(\mathrm{P}<0,01)$ das estimativas de MM em função do dia no qual a análise foi realizada (Tabela 10).

Tabela 8. Estimativas de componentes de variância do modelo destinado à avaliação individual de alimentos volumosos (Equação 4)

\begin{tabular}{|c|c|c|c|}
\hline Item & Estimativa & Valor relativo $(\%)$ & Valor-P \\
\hline & \multicolumn{3}{|c|}{ Feno } \\
\hline$\sigma_{\mathrm{L}}^{2}$ & 0,1557 & 58,2 & $<0,0001$ \\
\hline$\sigma_{\mathrm{D} / \mathrm{L}}^{2}$ & 0,0741 & 27,7 & $<0,0001$ \\
\hline Repetibilidade $\left(\sigma_{\varepsilon}^{2}\right)$ & 0,0378 & 14,1 & - \\
\hline Variância Total & 0,2676 & 100 & - \\
\hline Reprodutibilidade & 0,1935 & - & - \\
\hline $\mathrm{r} / \mathrm{R}^{1}$ & 0,1953 & - & - \\
\hline RH & 1,6763 & - & - \\
\hline \multirow[t]{2}{*}{ Média } & 7,47 & - & - \\
\hline & \multicolumn{3}{|c|}{ Cana-de-açúcar } \\
\hline$\sigma_{L}^{2}$ & 0,1757 & 43,6 & $<0,0001$ \\
\hline$\sigma_{\mathrm{D} / \mathrm{L}}^{2}$ & 0,0398 & 9,9 & 0,0346 \\
\hline Repetibilidade $\left(\sigma_{\varepsilon}^{2}\right)$ & 0,1873 & 46,5 & - \\
\hline Variância Total & 0,4028 & 100,0 & - \\
\hline Reprodutibilidade & 0,3630 & - & - \\
\hline $\mathrm{r} / \mathrm{R}^{1}$ & 0,5160 & - & - \\
\hline RH & 2,4021 & - & - \\
\hline \multirow[t]{2}{*}{ Média } & 7,05 & - & - \\
\hline & \multicolumn{3}{|c|}{ Silagem de Milho } \\
\hline$\sigma_{L}^{2}$ & 0,0425 & 39,3 & $<0,0001$ \\
\hline$\sigma_{\mathrm{D} / \mathrm{L}}^{2}$ & 0,0282 & 26,1 & $<0,0001$ \\
\hline Repetibilidade $\left(\sigma_{\varepsilon}^{2}\right)$ & 0,0374 & 34,6 & - \\
\hline Variância Total & 0,1081 & 100 & - \\
\hline Reprodutibilidade & 0,0799 & - & - \\
\hline $\mathrm{r} / \mathrm{R}^{1}$ & 0,4681 & - & - \\
\hline RH & 1,3458 & - & - \\
\hline Média & 5,62 & - & - \\
\hline
\end{tabular}

${ }^{1}$ Razão repetibilidade/reprodutibilidade. 
Rev. Bras. Saúde Prod. Anim., Salvador, v.18, n.1, p.62-75 jan./mar., 2017

Tabela 9. Estimativas de componentes de variância do modelo destinado à avaliação individual de alimentos concentrados (Equação 4)

\begin{tabular}{lccc}
\hline Item & Estimativa & Valor relativo (\%) & Valor-P \\
\hline & & Farelo de Soja & \\
\hline$\sigma_{\mathrm{L}}^{2}$ & 0,1047 & 56,6 & $<0,0001$ \\
$\sigma_{\mathrm{D} / \mathrm{L}}$ & 0,0504 & 27,2 & $<0,0001$ \\
Repetibilidade $\left(\sigma_{\varepsilon}^{2}\right)$ & 0,0300 & 16,2 & - \\
Variância Total & 0,1851 & 100,0 & - \\
Reprodutibilidade & 0,1347 & - & - \\
$\mathrm{r} / \mathrm{R}^{1}$ & 0,2227 & - & - \\
$\mathrm{RH}$ & 2,8382 & - & - \\
Média & 5,97 & - & - \\
\hline & & Milho & \\
\hline$\sigma_{\mathrm{L}}^{2}$ & 0,0174 & 33,5 & 0,0011 \\
$\sigma_{\mathrm{D} / \mathrm{L}}$ & 0,0235 & 45,3 & $<0,0001$ \\
Repetibilidade $\left(\sigma_{\varepsilon}^{2}\right)$ & 0,0110 & 21,2 & - \\
Variância Total & 0,0519 & 100,0 & - \\
Reprodutibilidade & 0,0284 & - & - \\
$\mathrm{r} / \mathrm{R}^{1}$ & 0,3873 & - & - \\
$\mathrm{RH}$ & 1,6671 & - & - \\
Média & 1,12 & - & $<0,0001$ \\
\hline & & Polpa Cítrica & $<0,0001$ \\
\hline$\sigma_{\mathrm{L}}^{2}$ & 0,8187 & 78,2 & - \\
$\sigma_{\mathrm{D} / \mathrm{L}}$ & 0,1522 & 14,5 & - \\
Repetibilidade $\left(\sigma_{\varepsilon}^{2}\right)$ & 0,0758 & 100,3 & - \\
Variância Total & 1,0467 & - & - \\
Reprodutibilidade & 0,8945 & - & - \\
$\mathrm{r} / \mathrm{R}^{1}$ & 0,0847 & - & - \\
$\mathrm{RH}$ & 3,9508 & - & \\
Média & 6,64 & & - \\
\hline
\end{tabular}

${ }^{1}$ Razão repetibilidade/reprodutibilidade.

Tabela 10. Nível descritivo de probabilidade para o erro tipo I associada à variação entre dias de análises para feno, cana-de-açúcar, silagem de milho (SM), farelo de soja (FS), milho, polpa cítrica (PC)

\begin{tabular}{ccccccc}
\hline \multirow{2}{*}{ Laboratório } & \multicolumn{7}{c}{ Material $^{1}$} \\
\cline { 2 - 7 } & Feno & Cana & SM & FS & Milho & PC \\
\hline 1 & 0,0095 & 0,0185 & 0,0740 & $<0,0001$ & 0,0180 & 0,4089 \\
2 & 0,2676 & 0,7313 & 0,8595 & 0,8466 & 0,7547 & 0,6707 \\
3 & $<0,0001$ & $<0,0001$ & 0,0372 & 0,0535 & 0,0006 & 0,0093 \\
4 & 0,1908 & 0,1319 & 0,2508 & 0,0144 & 0,0169 & 0,0265 \\
5 & $<0,0001$ & 0,0009 & 0,1376 & 0,0011 & 0,0420 & $<0,0001$ \\
6 & 0,0085 & 0,1318 & 0,0024 & 0,0012 & 0,6403 & 0,0435 \\
7 & $<0,0001$ & 0,4285 & $<0,0001$ & $<0,0001$ & $<0,0001$ & $<0,0001$ \\
\hline
\end{tabular}

A avaliação individual da variação intra-laboratorial (Figura 2), a qual constitui $o$ fator determinante da repetibilidade, indicou que, para alguns materiais, a variação entre dias de análise pôde representar de 0 (e.g., Figura 3, cana-de-açúcar, laboratório 2) a 98\% (e.g. Figura 3, milho, laboratório 7) da variação aleatória dos resultados. 

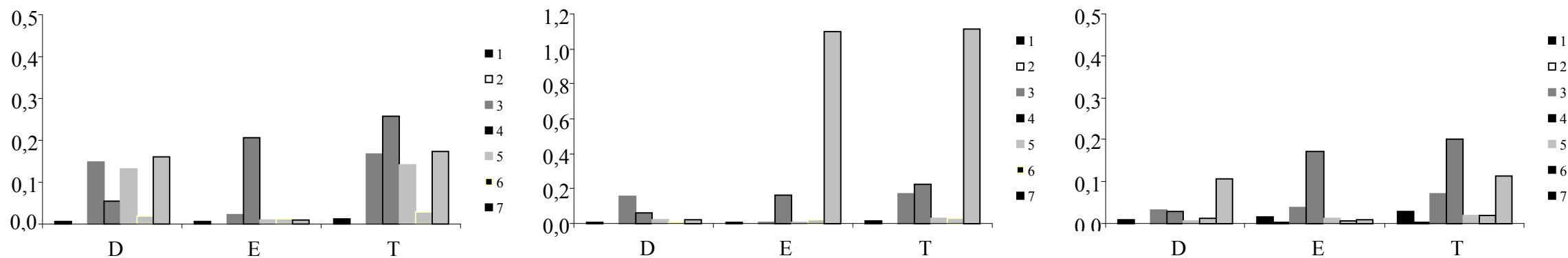

Milho

Farelo de Soja

Polpa Cítrica
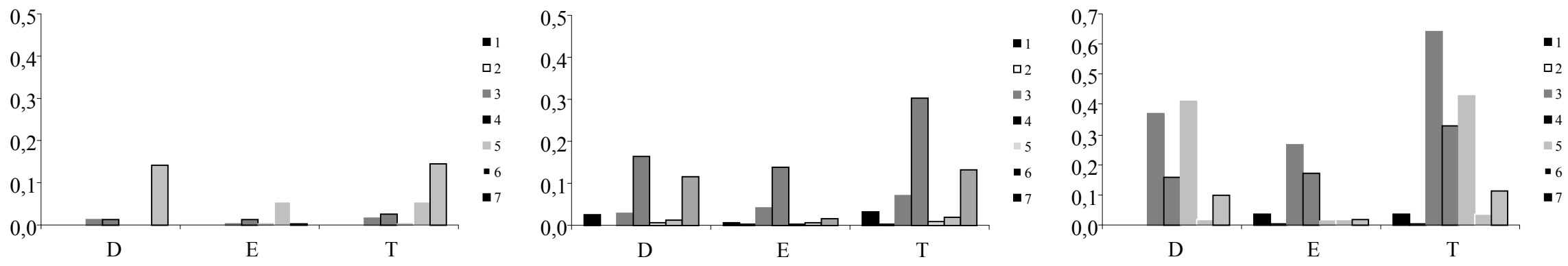

Figura 2. Avaliação das variâncias intra-laboratoriais $(\mathrm{D}=$ variância entre dias; $\mathrm{E}=$ variância do erro; $\mathrm{T}=$ variação aleatória total; $\mathrm{T}=$ $\mathrm{D}+\mathrm{E})$. 
Feno

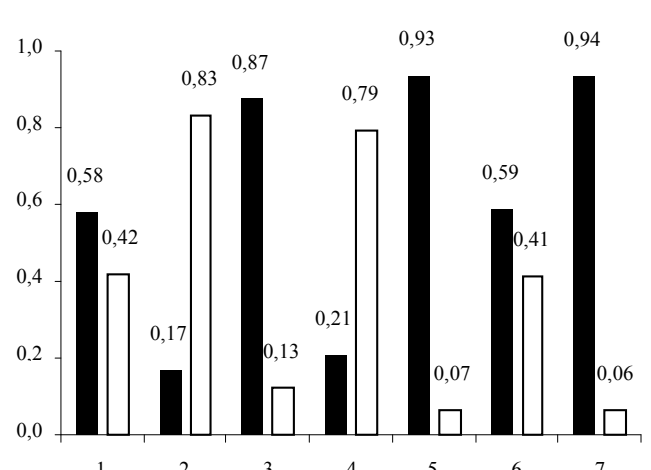

Cana-de-açúcar

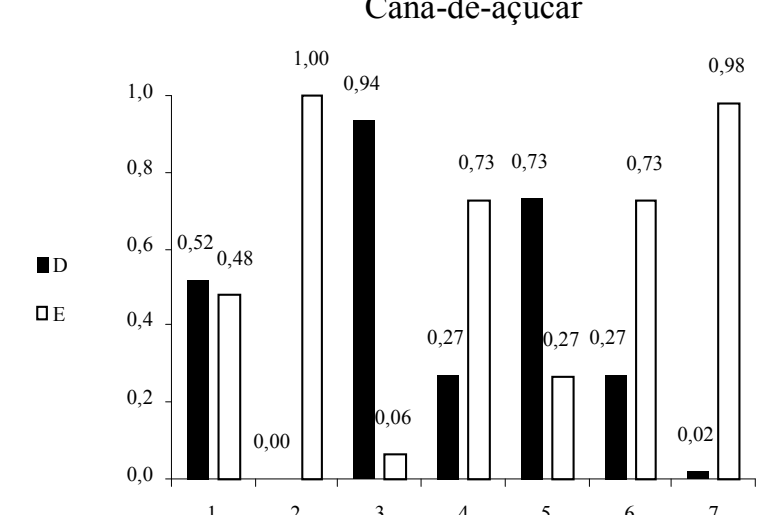

Silagem de Milho

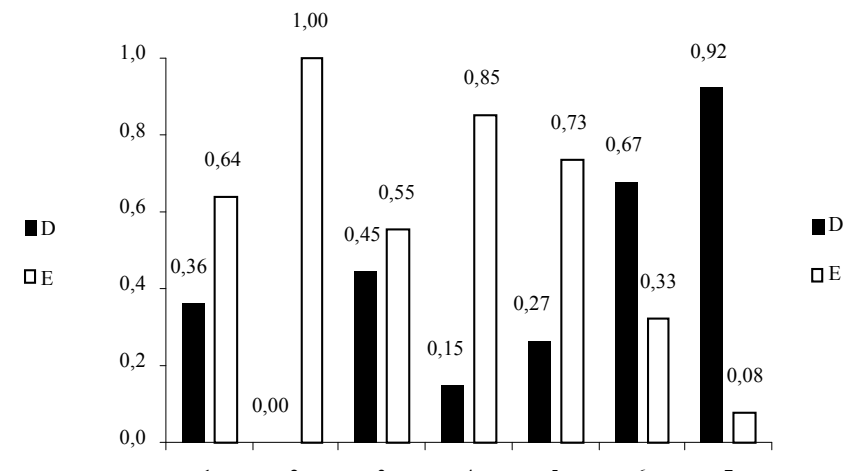

Milho

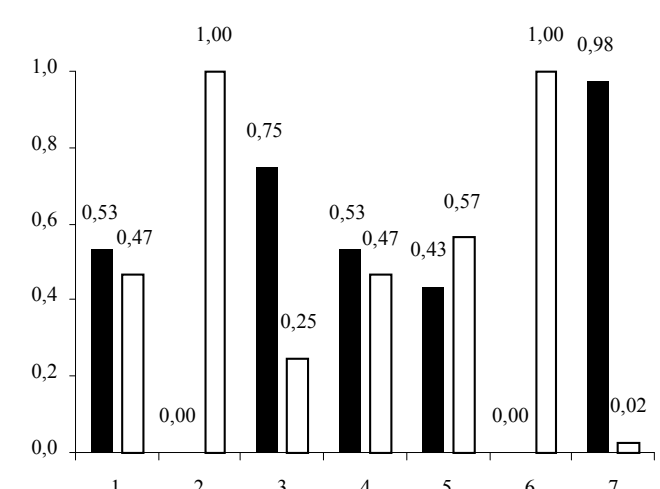

Farelo de Soja

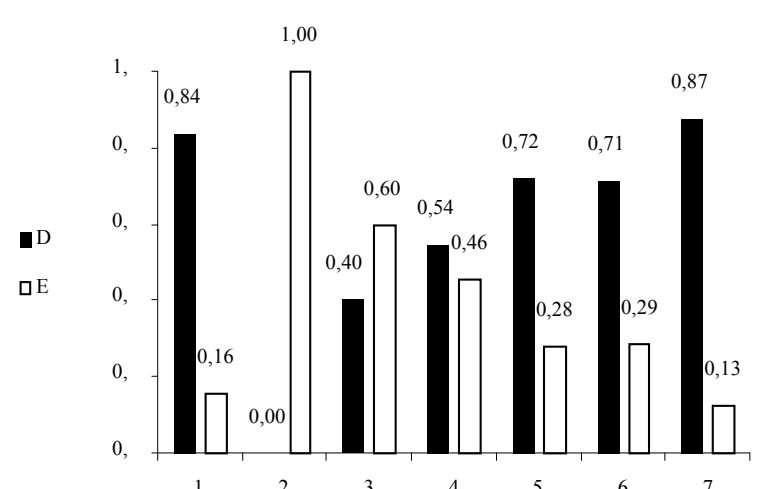

Polpa Cítrica

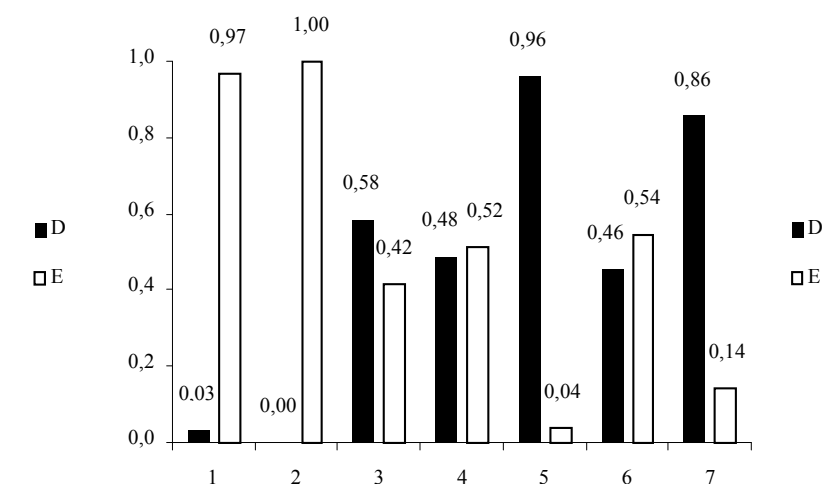

Figura 3. Avaliação fracional da composição da repetibilidade intra-laboratorial $(\mathrm{D}=$ contribuição da variância entre dias; $\mathrm{E}=$ contribuição da variância do erro). 
O comportamento verificado para algumas amostras torna evidente que os resultados obtidos no processo analítico foram diretamente dependentes do dia no qual a análise foi conduzida, o que obviamente é indesejável e pode atribuir efeito de confundimento sobre os teores de MM obtidos. Desta forma, foi evidenciado que problemas atribuídos à falta de padronização dos procedimentos intra-laboratoriais podem ser significativos sobre a precisão das estimativas.

As massas de amostras utilizadas nas avaliações variaram de 1 a 5 gramas (Tabela 11). Embora o método avaliado seja considerado robusto com relação a esta variável (SILVA \& QUEIROZ, 2002), a utilização de pequenas massas, como aquelas utilizadas pelos laboratórios 4, 5 e 6 , podem comprometer a exatidão devido a problemas na quantificação gravimétrica causados pelo pequeno resíduo de MM. As recomendações de massa seguem normalmente relação inversa com o teor de minerais na amostra (SILVA \& QUEIROZ, 2002); contudo, para a maioria dos materiais, massas próximas a $2 \mathrm{~g}$ proporcionaram boa sensibilidade nas avaliações. Adicionalmente, a avaliação das massas de amostras de forma relativa ao recipiente indicou que em alguns laboratórios podem estar sendo utilizadas amostras em demasia, o que pode retardar o processo de eliminação da matéria orgânica (Tabela 11).

Tabela 11. Características gerais do procedimento de avaliação de matéria mineral em função dos diferentes laboratórios

\begin{tabular}{|c|c|c|c|c|c|c|c|c|c|c|}
\hline \multirow{2}{*}{ Laboratório } & \multicolumn{10}{|c|}{ Característica $^{1}$} \\
\hline & MA & $\mathrm{MA} / \mathrm{A}$ & $\mathrm{MA} / \mathrm{V}$ & PPC & $\mathrm{CQ}$ & TPQ & TQ & PTT & $\mathrm{RM}$ & TRC \\
\hline 1 & 2 & 102 & 25 & LA, $105 / 1$ & $\mathrm{TF}$ & 600 & 3 & 1800 & $\mathrm{PA}$ & $\begin{array}{l}150- \\
200\end{array}$ \\
\hline 2 & $3-5$ & $239-398$ & $60-100$ & LA, $600 / 2$ & $\mathrm{TF}$ & 600 & 4 & 2400 & $\mathrm{~F}$ & $<200$ \\
\hline 3 & 2 & 102 & 23 & LHA, NI ${ }^{2}$ & $\mathrm{TF}$ & 590 & $\mathrm{NI}^{23}$ & $\mathrm{NI}^{2}$ & $\mathrm{~F}$ & 100 \\
\hline 4 & 1 & 63 & 16 & $\mathrm{NI}^{2}, 105 / 2$ & $\mathrm{TF}$ & 600 & 3 & 1800 & PA & 150 \\
\hline 5 & 1 & 80 & 20 & LA, $105 / 3$ & $\mathrm{TF}$ & 550 & 3 & 1650 & $\mathrm{~F}$ & $50-60$ \\
\hline 6 & 1 & 80 & 20 & LA, $105 / 4$ & $\mathrm{TF}$ & 600 & 4 & 2400 & $\mathrm{~F}$ & 150 \\
\hline 7 & $1,5-2$ & $119-159$ & $34-40$ & LA, $105 / 3$ & $\mathrm{TF}$ & 550 & 4 & 2200 & $\mathrm{~F}$ & $50-60$ \\
\hline
\end{tabular}

${ }^{1} \mathrm{MA}=$ massa de amostra $(\mathrm{g}) ; \mathrm{MA} / \mathrm{A}$ = relação entre a massa de amostra e a área de abertura do cadinho $\left(\mathrm{mg} / \mathrm{cm}^{2}\right) ; \mathrm{MA} / \mathrm{V}=$ relação entre a massa de amostra e o volume do cadinho $\left(\mathrm{mg} / \mathrm{cm}^{3}\right) ; \mathrm{PPC}=$ procedimentos de preparação do cadinho (LA, limpeza com água; LHA = limpeza com ácido e água; o binômio se refere ao procedimento de secagem do cadinho para obtenção das taras $-{ }^{\circ} \mathrm{C} / \mathrm{h}$ ); $\mathrm{CQ}=$ critério para estabelecimento do tempo de queima (TF, tempo fixo); TPQ = temperatura de queima $\left({ }^{\circ} \mathrm{C}\right) ; \mathrm{TQ}=$ tempo de manutenção na temperatura de queima $(\mathrm{h}) ; \mathrm{PTT}$, produto teórico entre a temperatura e o tempo de queima $\left({ }^{\circ} \mathrm{C} \times \mathrm{h}\right) ; \mathrm{RM}$, forma de resfriamento da mufla (F, fechada; PA, parcialmente aberta); TRC $=$ temperatura de retirada do cadinho da mufla.

${ }^{2} \mathrm{NI}$, característica não identificada.

${ }^{3} \mathrm{O}$ procedimento adotado na avaliação não pôde ser identificado, mas declarou-se que o tempo de queima varia em função do material analisado (forragens -4 horas; concentrados -6 horas; ossos -12 horas).

Embora em todos os laboratórios se utilize lavagem dos cadinhos previamente ao uso, os procedimentos de secagem são variados. Somente no laboratório 2 os cadinhos foram submetidos à calcinação para obtenção das taras (Tabela 11), o que 
é geralmente recomendado (CECCHI, 2003; CAMPOS et al., 2004), pois a deposição de pequenas impurezas de natureza orgânica podem incrementar o peso da tara, sendo contudo eliminadas durante a queima da amostra.

Em todos os laboratórios utiliza-se tempo fixo para a queima das amostras (Tabela 11), contudo as temperaturas de queima e o tempo de queima foram variados entre eles. As temperaturas utilizadas foram de acordo com recomendações encontradas na literatura, que vão de $550^{\circ} \mathrm{C}$ (THIEX \& NOVOTNY, 2012) a $600^{\circ} \mathrm{C}$ (WINDHAM, 1998; SILVA \& QUEIROZ, 2002). Entretanto, os tempos de queima foram iguais ou superiores às recomendações encontradas na literatura, as quais variam de 2 (WINDHAM, 1998; CECCHI, 2003) a 3 (CAMPOS et al., 2004; THIEX \& NOVOTNY, 2012) horas. No entanto, analisando-se de forma apenas heurística, o produto dado pela temperatura e pelo tempo de queima foi extremamente variado entre laboratórios (Tabela 11), indicando possível influência sobre a reprodutibilidade dos resultados.

Em dois dos laboratórios o resfriamento das amostras foi feito com abertura parcial da mufla, o que não é indicado, pois pode propiciar absorção de umidade pelas amostras localizadas próximas à abertura, principalmente se a temperatura de retirada for baixa. Adicionalmente, a abertura da mufla em altas temperaturas reduz a vida útil do equipamento por causar choques térmicos excessivos sobre o revestimento de cerâmica.

Em três laboratórios a retirada das amostras foi realizada em temperaturas consideradas baixas $\left(\leq 100^{\circ} \mathrm{C}\right)$, o que pode propiciar absorção de umidade e, consequentemente, distorção da massa de MM (Tabela 11). As temperaturas de retirada de amostras devem estar entre 150 e $200^{\circ} \mathrm{C}$. Em todos os laboratórios utiliza-se dessecador para resfriamento dos cadinhos.

Os teores de matéria mineral estimados pelos laboratórios participantes deste estudo apresentam baixa reprodutibilidade, o que é agravado pela presença de efeito de interação do material avaliado e do laboratório.

\section{AGRADECIMENTOS}

Ao INCT-Ciência Animal, ao CNPq, a CAPES e à FAPEMIG (PPM) pelo financiamento da pesquisa.

\section{REFERÊNCIAS}

\section{BARBIN, D. Componentes de}

variância: teoria e aplicações. 2.ed.

Piracicaba: FEALQ, 1993. 120p.

BRUTTEL, P.; SCHLINK, R. Water

determination by Karl Fischer

titration. Herisau: Metrohm, 2006. 80p.

CAMPOS, F.P.; NÚSSIO, C.M.B.; NÚSSIO, L.G. Métodos de análise de alimentos. Piracicaba: FEALQ, 2004. $135 \mathrm{p}$.

CECCHI, H.M. Fundamentos teóricos e práticos em análise de alimentos. 2 ed. Campinas: Unicamp, 2003. 207p.

HORWITZ, W.A.; ALBERT, R.; DEUTSCH, M. J.; THOMPSON, J. N. Precision parameters of methods of analysis required for nutrition labeling. Part I. Major nutrients. Journal of AOAC International, v.73, p.661-680, 1990. 
MERTENS, D.R. Challenges in measuring insoluble dietary fiber. Journal of Animal Science, v.81, p.3233-3249, 2003.

THIEX, N.; NOVOTNY, N.;

Determination of Ash in Animal Feed:

AOAC Official Method 942.05

Revisited. Journal of AOAC

International, v.95, p.1392-1397, 2012.

SILVA, D.J.; QUEIROZ, A.C. Análise de Alimentos: métodos químicos e biológicos. 3 ed. Viçosa, MG:

Universidade Federal de Viçosa, 2002. $235 \mathrm{p}$.

WINDHAM, W.R. Animal feed. In: ASSOCIATION OF OFFICIAL

ANALYTICAL CHEMISTS - AOAC (Ed.). Official Methods of Analysis of AOAC International. 16.ed. 4 rev. Gaithersburg: AOAC International, 1998.

Data de recebimento: 16/09/2015

Data de aprovação: 20/01/2017 